\title{
Immunogenicity of a 23-valent pneumococcal polysaccharide vaccine in Brazilian elderly
}

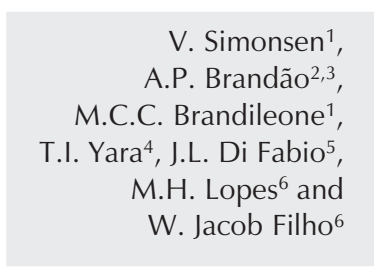

\author{
${ }^{1}$ Seção de Bacteriologia, Instituto Adolfo Lutz, São Paulo, SP, Brasil \\ ${ }^{2}$ Fundação Oswaldo Cruz, Rio de Janeiro, RJ, Brasil \\ ${ }^{3}$ Fundação Nacional de Saúde, São Paulo, SP, Brasil \\ ${ }^{4}$ Instituto Butantan, São Paulo, SP, Brasil \\ ${ }^{5}$ Organização Panamericana de Saúde, Washington, DC, USA \\ ${ }^{6}$ Faculdade de Medicina, Universidade de São Paulo, São Paulo, SP, Brasil
}

\section{Correspondence \\ V. Simonsen \\ Avenida Dr. Arnaldo, 355, 9o andar \\ 01246-902 São Paulo, SP \\ Brasil \\ E-mail: vsimonsen@ial.sp.gov.br}

Research supported by the Adolfo Lutz Institute, Brazil, and the Pan-American Health Organization.

Publication supported by FAPESP.

Received March 29, 2004

Accepted January 3, 2005

\begin{abstract}
Serum antibodies specific for the capsular polysaccharides of Streptococcus pneumoniae provide protection against invasive pneumococcal infection. In Brazil, this vaccine has been used for people over 65 years with clinical risk to develop pneumococcal infection since 1999 . We evaluated the immune response of 102 elderly subjects (75.5\% females and $24.5 \%$ males) with a mean age of 71 years, and 19 young healthy adults (63.2\% females and $36.8 \%$ males) with a mean age of 27 years. The elderly study group consisted of outpatients who received follow-up care in the Geriatric Department of General Hospital, Faculty of Medicine, University of São Paulo. None had acute illness at the time of vaccination. Both groups were immunized with one intra-deltoid injection with $0.5 \mathrm{ml}$ of a 23 -valent pneumococcal polysaccharide vaccine. The total IgG specific antibody concentrations to capsular polysaccharides $1,3,5,6 \mathrm{~B}, 8$, and 14 were determined against pre- and 1-month post-vaccination sera. All samples were analyzed according to the second-generation pneumococcal polysaccharide ELISA protocol. We observed that the pneumococcal polysaccharide vaccine evoked consistent antibody increase for serotypes $1,5,6 \mathrm{~B}, 8$, and 14 (geometric mean concentration increase of 2.46 in the elderly and 2.84 in the young adults). Otherwise, we observed no increase in antibody concentration for serotype 3 in both groups.
\end{abstract}

\section{Introduction}

Streptococcus pneumoniae is an important bacterial pathogen of children and adults worldwide, causing infection of the respiratory tract, bacteremia, and meningitis. Morbidity and mortality resulting from pneumococcal infection are high among the elderly,
Key words

- Streptococcus pneumoniae

- Vaccine

- Pneumococcal polysaccharide vaccine

- Immune response

- Elderly subjects who are at higher risk for invasive pneumococcal infections than the general population (1). Diseases caused by influenza and pneumococci, taken together, are the fifth leading cause of death for persons aged 65 years and older (2). The annual incidence of invasive pneumococcal disease has been estimated to be $\geq 50$ cases per 100,000 persons among the 
elderly in developed countries, whereas in Latin America and other developing countries the incidence is unknown $(1,3)$. Because of the high risk of morbidity and mortality associated with respiratory infections in the elderly, the World Health Organization recommends pneumococcal and influenza vaccination in this age group. The 23valent pneumococcal polysaccharide vaccine is about $50-80 \%$ effective in preventing invasive pneumococcal disease among older persons (4). In Brazil, this vaccine was introduced in a national campaign initiated in 1999, and is administered to institutionalized elderly subjects and to those recently hospitalized because of pneumonia $(5,6)$.

The aim of the present study was to assess the immune response of elderly persons immunized with the 23-valent pneumococcal vaccine. Specific antibodies to six pneumococcal capsular antigens that are among the major invasive serotypes for this age group were measured. In addition, we compared the immune response of this population with that of young healthy adults vaccinated at the same time.

\section{Material and Methods}

\section{Study group}

The study group consisted of 102 elderly outpatients who received follow-up care in the Geriatric Department of the General Hospital, Faculty of Medicine, University of São Paulo. The study was approved by the Ethics Committee and every participant signed the written informed consent. Seventy-seven subjects were females $(75.5 \%)$ with a mean age of 71 years (range: 61 to 87 years) and 25 were males (24.5\%) with a mean age of 72 years (range: 60 to 95 years). All outpatients had stable chronic illnesses and were monitored in a primary care clinic. None had acute illness at the time of vaccination or had previous vaccination against $S$. pneumoniae.

\section{Control group}

The control group consisted of 19 healthy young adults with a mean age of 27 years (range: 22 to 38 years). In this group there were 12 females $(63.2 \%)$ and 7 males $(36.8 \%)$. None had a history of pneumonia or previous vaccination with the 23 -valent vaccine.

\section{Immunization and serum collection}

Each subject $(\mathrm{N}=102+19)$ received one intra-deltoid injection with $0.5 \mathrm{ml}$ of the same lot of pneumococcal vaccine containing $25 \mu \mathrm{g}$ of the following serotypes: 1 to 5 , 6B, 7F, 8, 9N, 9V, 10A, 11A, 12F, 14, 15B, $17 \mathrm{~F}, 18 \mathrm{C}, 19 \mathrm{~A}, 19 \mathrm{~F}, 20,22 \mathrm{~F}, 23 \mathrm{~F}$, and 33F. Blood for serum analysis was obtained prior to immunization and 1 month after immunization. Serum samples were stored at $-70^{\circ} \mathrm{C}$ until the time for assay for capsule pneumococcal polysaccharide-specific IgG.

\section{Enzyme immunoassay (ELISA)}

The level of pneumococcal capsule specific IgG was measured with an international reference ELISA method $(7,8)$. Briefly, in this second-generation ELISA, microplates (Maxisorb, Nunc, Roskilde, Denmark) were coated with $100 \mu \mathrm{l}$ of a solution of pneumococcal polysaccharide (ATCC, Rockville, MD, USA). The plates were incubated at $37^{\circ} \mathrm{C}$ in a moist chamber for $5 \mathrm{~h}$. The subjects were evaluated for specific antibodies to six pneumococcal capsular polysaccharides (serotypes 1, 3, 5, 6B, 8, and 14). All serum samples were absorbed with purified pneumococcal cell wall polysaccharide (CPS) available from the Statens Seruminstitute (Copenhagen, Denmark) prior to the ELISA measurements. Sera were tested in duplicate and at two-fold serial dilutions. Specific antibodies were detected using polyclonal-peroxidase conjugate anti-human IgG gammachain specific raised in goat. 
Antibody concentrations are reported as $\mu \mathrm{g} / \mathrm{ml}$ calculated on the basis of the officially assigned $\mathrm{IgG}$ concentrations for $89-\mathrm{SF}$ reference serum. Antibody concentrations were calculated by comparison of the preand post-vaccination sera with 89-SF using a four-parameter logistic ELISA program (9). Two additional serum samples known to contain high and low levels of pneumococcal capsule specific antibodies were used as controls to ensure assay reproducibility.

\section{Statistical analysis}

Differences in log-transformed concentrations of capsule specific IgG antibodies between pre- and post-vaccination sera of both groups were calculated by analysis of variance for repeated measures. Correlation coefficients were calculated by the method of Pearson for linearity. Differences between groups in $\mathrm{IgG}$ increases and fold increases were determined by the Pearson chi-square and two-tailed Fisher exact tests. All P values were two-tailed and the level of significance was set at $\mathrm{P} \leq 0.05$. Statistics were calculated with the use of the Statistical Software for Windows (Stata Corporation, College Station, TX, USA).

\section{Results}

Prior to immunization, elderly and young adults had appreciable levels of specific IgG antibodies to the six serotypes analyzed. Both groups showed a wide variation in serum concentration of type-specific total IgG. As an example, the absolute concentration of serotype 14 specific antibody ranged from 2.3 to $120.2 \mu \mathrm{g} / \mathrm{ml}$ in the elderly and 2.1 to $36.0 \mu \mathrm{g} / \mathrm{ml}$ in the young adults. The geometric mean concentration (GMC) of the specific antibodies to the six serotypes in prevaccination sera varied among all subjects, ranging from 2.5 to $10.8 \mu \mathrm{g} / \mathrm{ml}$ in the elderly and from 1.7 to $9.8 \mu \mathrm{g} / \mathrm{ml}$ in the young adults (Figure 1). Before vaccination, the GMC of type-specific antibody to polysaccharide types 3,5 , and 6B was significantly higher in the elderly than in the young group. Among all polysaccharides analyzed, GMC to serotype 14 was the highest in pre-vaccination sera of both age groups (Figure 1). As a group, more than $90 \%$ of the elderly had more than $1 \mu \mathrm{g} / \mathrm{ml} \mathrm{IgG} \mathrm{specific} \mathrm{for} \mathrm{serotypes}$ $1,3,5$, and 8 , and all of them had at least this concentration for serotypes $6 \mathrm{~B}$ and 14 . In the young adult group, all subjects had $\geq 1 \mu \mathrm{g} / \mathrm{ml}$ $\operatorname{IgG}$ specific for serotypes 8 and 14 , and about $30 \%$ of them did not have $1 \mu \mathrm{g} / \mathrm{ml} \mathrm{IgG}$ antibody to serotypes 3 and 5 . The young adult group presented the lowest percentage of subjects $(68 \%)$ with an $\mathrm{IgG}$ concentration of less than $1 \mu \mathrm{g} / \mathrm{ml}$ in pre-immunization sera against serotype 3 (Table 1 ).

Following immunization, all subjects had a significant increase in antibody responses to the serotypes analyzed, with the exception of type $6 \mathrm{~B}$ for the young adult group and type 3 for both groups (Figure 1). We also observed wide variation in the concentration of type-specific total IgG elicited. For example, the absolute concentration of serotype 14 specific antibodies ranged from 4.7

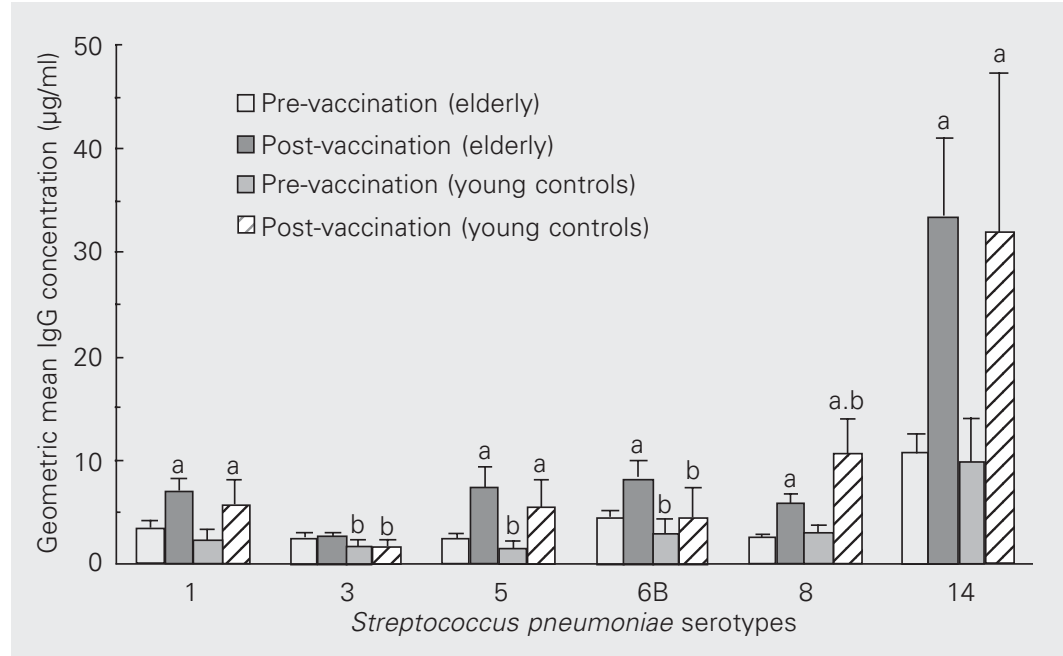

Figure 1. Geometric mean concentration and $95 \%$ confidence intervals of IgG specific for pneumococcal capsular polysaccharide in serum samples from 102 elderly and 19 young adults, pre- and post-vaccination. ap $<0.05$ for pre-vaccination sera compared to postvaccination sera; ${ }^{\text {bP }}<0.05$ for young adults compared to elderly subjects (analyses of variance for repeated measures). 
to $952.8 \mu \mathrm{g} / \mathrm{ml}$ in the elderly and from 3.6 to $112.7 \mu \mathrm{g} / \mathrm{ml}$ in the young adults. Specific antibody GMC increased after vaccination by more than $3 \mu \mathrm{g} / \mathrm{ml}$ for five of the six serotypes analyzed in the elderly and for four of the six serotypes in the young adults. Serotype 14 elicited the highest GMC and the highest absolute increase in total antibody concentrations (more than $20 \mu \mathrm{g} / \mathrm{ml}$ ) for both groups (Figure 1).

Having established that pneumococcal vaccination generated a specific and appreciable response against five serotypes in both age groups, we determined whether the immune response of the elderly differed from that of young people. In the elderly group, the post-vaccination GMCs were higher to serotypes $1,3,5,6 \mathrm{~B}$, and 14 , with $\mathrm{P}<0.05$ for serotypes 3 and $6 \mathrm{~B}$. On the other hand, the younger controls developed a significantly higher humoral response to serotype 8 , although both groups had similar baseline responses to this serotype (Figure 1). The fold increases in serotype-specific IgG against serotypes $1,3,5,6 \mathrm{~B}$, and 14 were comparable between the two groups (Table 1). The young adults, however, had a significantly higher fold increase against serotype 8 . The mean increase in type-specific antibody after vaccination was greater than two-fold for serotypes 1, 5, 8, and 14 in both groups. Serotypes 3 and $6 \mathrm{~B}$ elicited lower increases

Table 1. Geometric mean concentration (GMC) and increases of IgG antibodies to 6 polysaccharides of Streptococcus pneumoniae in elderly (N = 102) and young adults $(\mathrm{N}=19)$.

\begin{tabular}{|c|c|c|c|c|c|c|c|c|c|c|}
\hline & \multicolumn{2}{|c|}{$\mathrm{GMC} \mu \mathrm{g} / \mathrm{ml}(95 \% \mathrm{Cl})$} & \multicolumn{2}{|c|}{$\%$ with $\lg G \geq 1 \mu g$} & \multicolumn{2}{|c|}{$\%$ with $\mathrm{FI} \geq 2$} & \multicolumn{2}{|c|}{$\%$ with $\mathrm{FI} \geq 4$} & \multicolumn{2}{|c|}{$\%$ with $\lg G \geq 1 \mu$ increase } \\
\hline & Elderly & $\begin{array}{l}\text { Young } \\
\text { adults }\end{array}$ & Elderly & $\begin{array}{l}\text { Young } \\
\text { adults }\end{array}$ & Elderly & $\begin{array}{l}\text { Young } \\
\text { adults }\end{array}$ & Elderly & $\begin{array}{l}\text { Young } \\
\text { adults }\end{array}$ & Elderly & $\begin{array}{l}\text { Young } \\
\text { adults }\end{array}$ \\
\hline \multicolumn{11}{|l|}{ Serotype 1} \\
\hline Pre-vaccination & $3.5(3.0-4.1)$ & $2.3(1.6-3.4)$ & 92 & 89 & & & & & & \\
\hline 1 month & $7.1(6.1-8.2)^{a}$ & $5.7(3.9-8.3)^{a}$ & 99 & 100 & 40 & $68^{b}$ & 18 & 11 & 66 & 79 \\
\hline Fold increase & 2.0 & 2.4 & & & & & & & & \\
\hline \multicolumn{11}{|l|}{ Serotype 3} \\
\hline Pre-vaccination & $2.6(2.3-3.0)$ & $1.7(1.2-2.3)^{\mathrm{b}}$ & 98 & $68^{b}$ & & & & & & \\
\hline 1 month & $2.7(2.3-3.0)$ & $1.7(1.2-2.3)^{\mathrm{b}}$ & 97 & $74^{b}$ & 1 & 0 & 1 & 0 & 9 & 0 \\
\hline Fold increase & 1.02 & 1.0 & & & & & & & & \\
\hline \multicolumn{11}{|l|}{ Serotype 5} \\
\hline Pre-vaccination & $2.5(2.2-2.9)$ & $1.6(1.1-2.1)^{b}$ & 93 & $74^{b}$ & & & & & & \\
\hline 1 month & $7.5(6.1-9.3)^{\mathrm{a}}$ & $5.4(3.5-8.3)^{a}$ & 99 & 100 & 50 & 53 & 32 & 42 & 70 & 58 \\
\hline Fold increase & 3.0 & 3.5 & & & & & & & & \\
\hline \multicolumn{11}{|l|}{ Serotype 6B } \\
\hline Pre-vaccination & $4.4(3.9-5.0)$ & $3.0(2.1-4.3)^{\mathrm{b}}$ & 100 & 95 & & & & & & \\
\hline 1 month & $8.2(6.8-9.9)^{a}$ & $4.4(2.6-7.5)^{\mathrm{b}}$ & 100 & 95 & 38 & 16 & 15 & 11 & 62 & $37 \mathrm{~b}$ \\
\hline Fold increase & 1.9 & 1.5 & & & & & & & & \\
\hline \multicolumn{11}{|l|}{ Serotype 8} \\
\hline Pre-vaccination & $2.5(2.3-2.8)$ & $2.9(2.2-3.8)$ & 97 & 100 & & & & & & \\
\hline 1 month & $5.9(5.1-6-9)^{a}$ & $10.6(8.2-13.8)^{a, b}$ & 99 & 100 & 56 & 75 & 23 & 50 & 76 & 92 \\
\hline Fold increase & 2.3 & $3.6^{b}$ & & & & & & & & \\
\hline \multicolumn{11}{|l|}{ Serotype 14} \\
\hline Pre-vaccination & $10.8(9.3-12.5)$ & $9.8(6.9-14.1)$ & 100 & 100 & & & & & & \\
\hline 1 month & $33.4(27.2-40.9)^{a}$ & $31.9(21.4-47.3)^{a}$ & 100 & 100 & 67 & 63 & 33 & 37 & 90 & 95 \\
\hline Fold increase & 3.1 & 3.2 & & & & & & & & \\
\hline
\end{tabular}


$(<2)$ in both age groups.

Because GMC and mean fold increase may not accurately indicate differences in immune responses between the two populations, we compared the proportion of elderly and young adults who achieved at least twofold and four-fold increases in antibody levels against the serotypes analyzed. Vaccination produced a two-fold rise in specific antibody in $50 \%$ or more of all subjects against serotypes 5,8 , and 14 , with comparable proportions of elderly and young adult subjects. More young adults $(68 \%)$ had a two-fold response to serotype 1 than the elderly (40\%). Although type 6B is considered to be a poor immunogen (10), $38 \%$ of elderly subjects had two-fold increases in specific antibody after vaccination and only $16 \%$ of young subjects could set up this response. The proportion of subjects achieving a four-fold response was similar for the two age groups: more than $30 \%$ of all subjects presented this increase for serotypes 5 , and 14 and less than $18 \%$ of all subjects could set up a four-fold response to serotypes 1 and 6B. Although we observed a considerable proportion of young adults $(50 \%)$ that set up a four-fold rise to serotype 8 , the response was not significantly different between the two age groups (Table 1).

As an arbitrary estimate of protective concentrations of pneumococcal capsular antibodies, we analyzed the cut-off value of $1 \mu \mathrm{g} / \mathrm{ml}$, as usually done for anticapsular antibodies to Haemophilus influenzae type b $(11,12)$. After vaccination, almost all elderly subjects achieved at least $1 \mu \mathrm{g} / \mathrm{ml}$ of total IgG specific for all six serotypes analyzed. Among the young adults, we also observed a good response, all of them achieving a $\geq 1$ $\mu \mathrm{g} / \mathrm{ml}$ response to serotypes 1 and 5 . The lowest percentage (74\%) was observed in response to serotype 3 (Table 1). When all six serotypes were analyzed, vaccination increased the overall average of the elderly with an antibody concentration $\geq 1 \mu \mathrm{g} / \mathrm{ml}$ from 97 to $99 \%$ and this increase varied from
88 to $95 \%$ in the young adult group.

When the immune response was assessed for a type-specific IgG increase $\geq 1 \mu \mathrm{g} / \mathrm{ml}$ after vaccination, more than $60 \%$ of all elderly subjects showed this increase in response to all serotypes, except type 3 . In both groups, the lowest percentage of subjects with an IgG increase $\geq 1 \mu \mathrm{g} / \mathrm{ml}$ was observed for serotype 3 . A statistically significant difference between the two age groups was seen for serotype $6 \mathrm{~B}$, in response to which the young adults had a significant lower percentage (37\%) than the elderly subjects $(62 \%)$ who achieved this increase (Table $1)$.

We also evaluated the number of serotypes for which each subject achieved an increase $\geq 1 \mu \mathrm{g} / \mathrm{ml}$ and a two-fold rise in IgG concentration after vaccination (Figure 2). The elderly and the young adults had comparable frequencies of these immune response parameters. None of the subjects achieved a two-fold response to all six serotypes simultaneously, but $7 \%$ of the elderly had an increase $\geq 1 \mu \mathrm{g} / \mathrm{ml}$ for the six antigens. When five serotypes were analyzed, $9 \%$ of the elderly and $8 \%$ of the young adults achieved a two-fold response, and about $25 \%$ of all

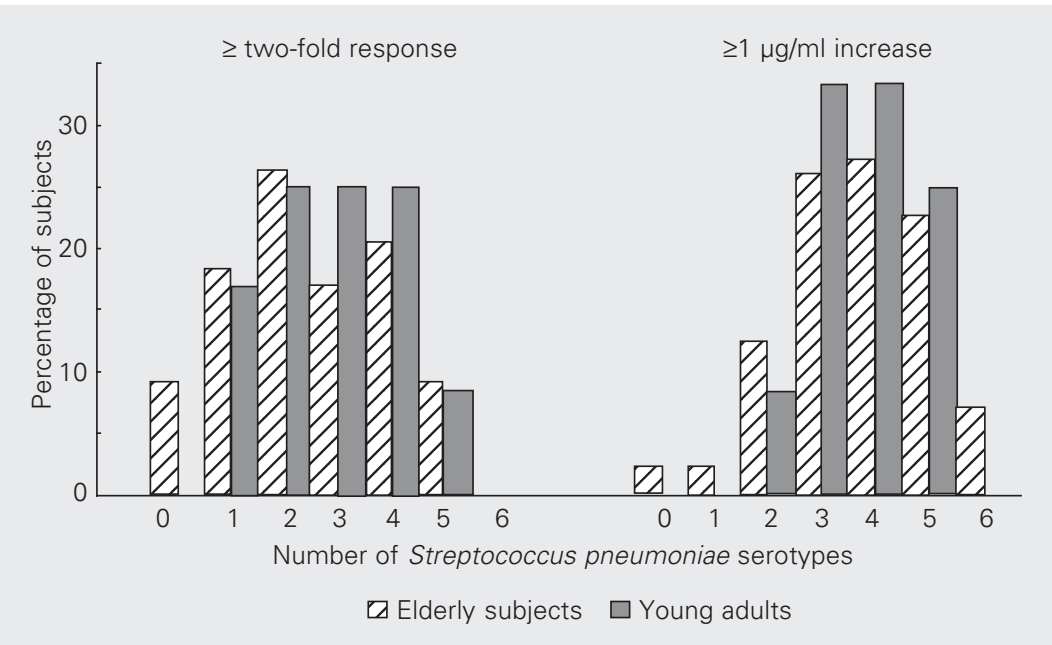

Figure 2. Immune response after pneumococcal vaccination reported as a two-fold increase or increase of $1 \mu \mathrm{g} / \mathrm{ml}$ or more of polysaccharide-specific lgG against the indicated number of serotypes. No significant differences were detected between elderly and young adults ( $P>0.05$, chi-square test). 
subjects had an increase $\geq 1 \mu \mathrm{g} / \mathrm{ml}$. Among the elderly, 9 and $2 \%$, respectively, did not achieve a two-fold response or an increase $\geq 1 \mu \mathrm{g} / \mathrm{ml}$ for any of the six serotypes. In the younger group we did not detect a null response.

In an attempt to determine if there was a relationship between specific antibody concentrations for any two serotypes, we performed all pairwise comparisons of antibody concentrations for both age groups, as suggested by Coughlin et al. (13). In Figure 3, we present the correlation coefficients for pre- and post-immunization sera obtained for each group. In pre-immunization sera, the comparison of each elderly subject's antibody level by cluster analysis revealed a considerable correlation $(r \geq 0.57)$ between the concentrations of specific $\operatorname{IgG}$ for serotypes $1,3,5,6 \mathrm{~B}$, and 8 . Serotype 14 correlated distinctly more poorly $(\mathrm{r}<0.26)$ with the other serotypes. In the analysis of postimmunization sera, the correlation coefficients decreased for all pairwise comparisons of type-specific antibody concentration, with the exception of serotype 14 . We obtained similar results in the young adult group.

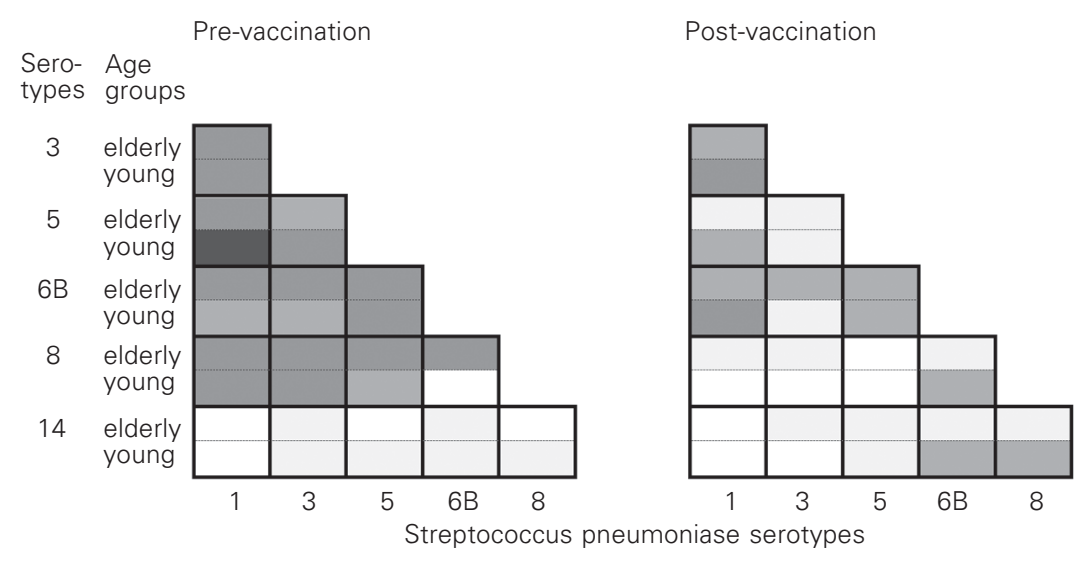

Patterns for correlation coefficient ranges \begin{tabular}{|l|l|l|l|l|}
$\leq 0.2$ & $>0.2$ & $>0.4$ & $>0.6$ & $>0.8$ \\
\hline
\end{tabular}

Figure 3. Correlation coefficients for antibody concentration between pairs of pneumococcal capsular polysaccharides in pre- and post-vaccination sera from 102 elderly subjects and 19 young adults. Degrees of correlations are indicated by shading patterns.

\section{Discussion}

Protection after immunization is thought to be dependent upon the production of circulating pneumococcal type-specific antibodies that act alone or in concert with complement proteins to opsonize bacteria and promote their phagocytosis. Antibodies specific for the capsular polysaccharide of $S$. pneumoniae are an important part of the human host defense, protecting against invasive pneumococcal disease. Consequently, the measurement of antibodies specific for pneumococcal polysaccharides is important in estimating vaccine-induced immune protection.

We measured type-specific antibodies against 5 pneumococcal polysaccharide antigens $(1,3,5,6 \mathrm{~B}$, and 14$)$ that belong to the most common serotypes causing invasive infections in the elderly in Brazil (14). Although serotype 8 is less common in Brazil, this antigen was included because, like serotypes 2,9 , and 14 , it has reproducible immunogenicity as defined by a two-fold increase in antibody titers obtained for normal controls $(15,16)$. All six serotypes analyzed in the present study are included in the 23valent vaccine.

ELISA has been widely used to measure immunity to pneumococci and the immunogenicity of pneumococcal vaccines. Correlation between this method and opsonophagocytic (functional) activity has been considered reasonably good in post-immunization serum samples from adults and infants (1719).

When we initiated the immunoassays in the late 90's we used the second-generation standardization of pneumococcal ELISA. This protocol was recommended by the World Health Organization at that time and was extensively used by experts $(7,8,20)$. The pneumococcal capsular polysaccharide preparations used in the present ELISA are contaminated with a common cell wall (CPS), and therefore antibodies to C-PS should 
be absorbed to improve the specificity of the ELISA (21). However, recent reports have shown that the second-generation ELISA was found to have insufficient specificity when serum samples from unimmunized subjects were analyzed (13). Furthermore, several investigators reported that, despite the C-PS absorption, antibodies cross-reactive with several types of pneumococcal polysaccharides are still measured $(17,18,22)$. As reported elsewhere $(17,18,23)$, cross-reactive antibodies are found more often in the sera of non-immunized infants and adults than in the sera of infants or adults immunized with pneumococcal vaccines, suggesting that the majority of the antibodies induced by vaccination are polysaccharide type or group specific. At the present time a thirdgeneration assay format has been adopted by experts (24) and an additional step is performed for the removal of cross-reactive antibodies. This additional absorption step, using $22 \mathrm{~F}$ polysaccharide antigen, improves the correlation between ELISA and opsonophagocytic activity, the two methodologies widely used to characterize the immune response (25).

In our analysis, the pre-vaccination sera of the majority of elderly and young adults had detectable levels of antibody for the six polysaccharides analyzed in pre-vaccination sera. Considering the limitation of the ELISA used in our study, this measurement may reflect antibodies cross-reactive with several types of pneumococcal polysaccharides, antibodies acquired during life as a result of colonization, previous infection with S. pneumoniae, or other cross-reactive organisms. Nevertheless, the basal anti-specific capsular polysaccharide IgG concentrations found in our study were in agreement with previous reports on elderly people from industrialized countries (26) analyzed by second-generation ELISA.

In general, the elderly and the young adults developed a significant mean increase of antibody levels for 5 of 6 and 4 of 6 capsular polysaccharides, respectively. The present study demonstrated that, at 1 month after immunization, the sera of elderly persons had levels of specific capsular antibodies similar to those of healthy younger persons, as reported elsewhere $(16,27)$. For both groups, serotypes 3 and 6B elicited lower increases $(<2)$ and, by this criterion, these antigens appeared to be less immunogenic in both groups $(10,26)$. The finding that most elderly subjects responded satisfactorily to the pneumococcal vaccine agrees with the results of other studies (27-29). It is still possible that healthy elderly adults, such as those in our study, respond normally to pneumococcal polysaccharide antigens, but that their response is reduced with the ill effects of aging and the increase of co-morbid conditions. As reported elsewhere (20), the response to certain polysaccharides may be reduced, with a more marked reduction in persons $>85$ years of age. In our study we found that serotype 3 was poorly immunogenic, eliciting the lowest absolute IgG concentration and fold increase in specific antibodies for both groups. In a similar serumconversion study, Rubbins et al. (26) observed that serotypes 3 and 4 account for many of the vaccine failures seen in clinical trials, suggesting that future vaccines for use in the elderly must be improved for these antigens.

Although Sorensen et al. (30) noted that subjects with high pre-immunization antibody concentrations tend to have smaller increases than those with low pre-immunization concentrations, we found that serotype 14 appeared to be one of the most immunogenic by rise and absolute rise criteria, though this serotype had the highest baseline level. These results were similar to those reported by others $(12,26)$. We also observed that serotypes 1,5 , and 8 induced a high level of humoral response for both groups. As also noted by other investigators $(15,16)$, serotype 8 induced the highest fold increase in the young adult subjects. Actu- 
ally, the concentration of anti-polysaccharide $8 \mathrm{IgG}$ in 89 -SF serum had not been assigned at the time of our study, and therefore we performed the cross-standardization method of Conception and Frasch (31). The assigned IgG antibody value for serotype 8 defined in our study did not differ from that found later by Quataert et al. (32). We confirmed the usefulness of the cross-standardization method to calibrate unknown IgG concentrations to pneumococcal serotypes.

It has been often stated that a normal antibody response to vaccination should include at least a two-, three-, or four-fold increase in antibody titer to the antigens analyzed. Traditional criteria for the interpretation of the immune response to pneumococcal polysaccharide have been proposed based on the difference between the postand pre-immunization antibody concentrations regardless of the increase after immunization $(15,30)$. In the present study, the percentage of vaccinees attaining a two-fold rise in IgG antibody was similar for the two age groups for 5 of the 6 serotypes analyzed. Only for serotype 1 was the percentage of elderly subjects that achieved a two-fold response lower than that of young adults. It should be noted that some investigators found a lower percentage of institutionalized elderly subjects who responded to serotypes 6B and 14 (20). When we compared the fourfold rise in specific IgG antibody concentration for the two groups, the percentage of young adult and elderly subjects was similar for all serotypes.

In a study of post-vaccinal immune response in the elderly, Rubbins et al. (26) concluded that only $3.7 \%$ of the elderly had two-fold increases in antibody for all 23 serotypes, $48 \%$ had two-fold increases for 15 or more serotypes, and $80 \%$ responded to 6 or more vaccinal serotypes. In our study we could not find in either group subjects who set up simultaneously a two-fold immune response against the 6 pneumococcal capsular antigens analyzed. Although some investigators try to characterize the poor responders $(16,26)$, we were not able to identify them due the small number of polysaccharides analyzed.

As reported elsewhere $(13,26)$, we observed that pre-vaccination levels of antibodies to serotypes $1,3,5,6 \mathrm{~B}$, and 8 were considerably correlated in the elderly. As discussed earlier, this event may be observed when the assay is not specific, and may be due to an antigenic stimulus common to these serotypes, most likely a cross-reactive antigen. On the other hand, serotype 14 showed the lowest correlation coefficient, confirming the considerably type-specific measurement of antibodies to serotype 14 by ELISA demonstrated in several studies $(13,17,22,23,25)$. However, after vaccination, all pairwise correlations showed a consistent decrease, with the exception of serotype 14 . These data suggest an improvement in antibody specificity to serotypes $1,3,5$, $6 \mathrm{~B}$, and 8 . As reported by others $(13,23,26)$, lower correlation coefficients for antibody concentration with other serotypes could mean the absence of cross-reactive antigens. The increase of correlation for serotype 14 in post-vaccinal sera may suggest an overall increase in total $\mathrm{IgG}$ concentration. We observed similar correlations in the pre- and post-vaccination sera of young adults.

We confirmed that pneumococcal vaccination raised the levels of antibodies against five of the six pneumococcal serotypes in the elderly, and elicited a relevant immune response in both age groups. The 23-valent polysaccharide vaccine may induce a protective immune response against invasive pneumococcal diseases in the elderly population. Our study demonstrated that the mean increase in type-specific antibody after vaccination was greater than two-fold for serotypes $1,5,8$, and 14 in both groups, even though this calculation was influenced by baseline concentrations obtained by secondgeneration ELISA. In fact, for five of six polysaccharides analyzed, our study may 
have overestimated the $\mathrm{IgG}$ concentration in pre-immunization sera, since the second-generation ELISA we performed may not be optimal for measuring type-specific antibodies, especially in non-immune serum samples. Our laboratory is currently using the protocol recommended for the third-generation pneumococcal antibody ELISA (http:/ /www.vaccine.uab.edu).

\section{Acknowledgments}

We gratefully acknowledge the constructive criticism of Dr. Moon H. Nahm, University of Alabama, Birmingham, USA, Dr. Carl E. Frasch, FDA, USA, for kindly providing reference serum 89-SF and Dr. Luiz Jacintho da Silva, Coordenação dos Institutos de Pesquisa, Secretaria de Estado da Saúde de São Paulo, for the support of this publication.

\section{References}

1. Vlasich C (2001). Pneumococcal infection and vaccination in the elderly. Vaccine, 19: 2233-2237.

2. Mieczkowisk TA \& Wilson SA (2002). Adult pneumococcal vaccination: a review of physician and patients. Vaccine, 20: 1383-1392.

3. Fedson DS, Scott JA \& Scott G (1999). The burden of pneumococcal disease among adults in developed and developing countries: what is and is not known. Vaccine, 17: S11-S18.

4. Fedson DS (1999). The clinical effectiveness of pneumococcal vaccination: a brief review. Vaccine, 17: S85-S90

5. Toniolo-Neto J, Weckx LW, Halker E, Lopes CH, de Menezes Succi RC, de Paiva TM, Ishida MA \& Forleo-Neto E (1999). Safety of simultaneous pneumococcal and influenza vaccination in elderly patients in Brazil. Drugs and Aging, 15 (Suppl 1): 43-45

6. Aranda CMSS (1999). Vacinação para o idoso no ano de 1999. Revista CIP - Ações em Saúde, 3: 13-18.

7. Plikaytis BD, Goldblat D, Frasch CE et al. (2000). An analytical model applied to a multicenter pneumococcal enzyme linked immunosorbent assay study. Journal of Clinical Microbiology, 38: 2043-2050.

8. Quataert SA, Kirch CS, Quackenbush LJ, Phipps DC, Strohmeyer S, Cimino CO, Skuse J \& Madore DV (1995). Assignment of weightbased antibody units to a human antipneumococcal standard reference serum, lot 89-S. Clinical and Diagnostic Laboratory Immunology, 2: 590-597.

9. Plikaytis BD, Holder PF \& Carlone GM (1995). Program ELISA for Windows User's Manual, Version 1.00. Centers for Disease Control and Prevention, Atlanta, GA, USA.

10. Shelley MA, Jacoby H, Riley GJ, Graves BT, Pichichero M \& Treanor JJ (1997). Comparison of pneumococcal polysaccharide and CRM197 conjugated pneumococcal oligosaccharide vaccines in young and elderly adults. Infection and Immunity, 65: 242-247.

11. Kayhty H, Ahman H, Ronnberg PR, Tillikainen R \& Eskola J (1995). Pneumococcal polysaccharide-meningococcal outer membrane protein complex conjugate vaccine is immunogenic in infants and children. Journal of Infectious Diseases, 172: 1273-1278.

12. Sankilampi U, Honkanen PO, Bloigu A, Herva E \& Leinonen M (1996). Antibody response to pneumococcal capsular polysaccharide vaccine in the elderly. Journal of Infectious Diseases, 173: 387393.

13. Coughlin RT, White AC, Anderson CA, Carlone GM, Klein DL \& Treanor J (1998). Characterization of pneumococcal specific antibodies in healthy unvaccinated adults. Vaccine, 16: 1761-1767.

14. Brandileone MC, Andrade AL, Di Fabio JL, Guerra ML \& Austrian R (2003). Appropriateness of a pneumococcal conjugate vaccine in
Brazil: potential impact of age and clinical diagnosis, with emphasis on meningitis. Journal of Infectious Diseases, 187: 1206-1212.

15. Go ES \& Ballas ZK (1996). Anti-pneumococcal antibody response in normal subjects: a meta analysis. Journal of Allergy and Clinical Immunology, 98: 205-215.

16. Rubbins JB, Puri AKG, Loch J, Charboneau D, MacDonald R, Opstad $N$ \& Janoff EM (1998). Magnitude, duration, quality and function of pneumococcal vaccine responses in elderly adults. Journal of Infectious Diseases, 178: 431-440.

17. Soininen A, van den Dobbelsteen G, Oomen L \& Kayhty H (2002). Are the enzyme immunoassays for antibodies to pneumococcal capsular polysaccharides serotype specific? Clinical and Diagnostic Laboratory Immunology, 7: 468-476.

18. Soininen A, Karpala M, Wahlman SL, Lehtonen H \& Kayhty H (2002). Specificities and opsonophagocytic activities of antibodies to pneumococcal capsular polysaccharides in sera of unimmunized young children. Clinical and Diagnostic Laboratory Immunology, 9: 10321038.

19. Romero-Steiner S, Libutti D, Pais LB, Dykes J, Anderson P, Whitin JC, Keyserling HL \& Carlone GM (1997). Standardization of an opsonophagocytic assay for the measurement of functional antibody activity against Streptococcus pneumoniae using differentiated HL-60 cells. Clinical and Diagnostic Laboratory Immunology, 4 415-422.

20. Romero-Steiner S, Musher DM, Cetron MS, Pais LB, Groover JE, Fiore AE, Plykaytis BD \& Carlone G (1999). Reduction in functional antibody activity against Streptococcus pneumoniae in vaccinated elderly individuals highly correlates with decreased IgG antibody avidity. Clinical Infectious Diseases, 29: 281-288.

21. Koskella M (1987). Serum antibodies to pneumococcal C polysaccharide in children: response to acute pneumococcal otites media or to vaccination. Pediatric Infectious Disease, 6: 519-526.

22. Yu X, Sun Y, Frasch C, Conception N \& Nahm MH (1999). Pneumococcal capsular polysaccharide preparations may contain non-C polysaccharide contaminants that are immunogenic. Clinical and Diagnostic Laboratory Immunology, 6: 519-524.

23. Soininen A, Pursiainen H, Kilpi T \& Kayhty H (2001). Natural development of antibodies to pneumococcal polysaccharide depends on the serotype: association with pneumococcal carriage and acute otitis media in young children. Journal of Infectious Diseases, 184: 569-575.

24. Wernette CM, Frasch CE, Madore DV et al. (2003). Enzyme-linked immunosorbent assay for quantification of human antibodies to 
pneumococcal polysaccharides. Clinical and Diagnostic Laboratory Immunology, 10: 514-519.

25. Conception N \& Frasch C (2001). Pneumococcal type 22 F polysaccharide absorption improves the specificity of a pneumococcalpolysaccharide enzyme-linked immunosorbent assay. Clinical and Diagnostic Laboratory Immunology, 8: 266-272.

26. Rubbins JB, Alter M, Loch J \& Janoff EN (1999). Determination of antibody responses of elderly adults to all 23 capsular polysaccharides after pneumococcal vaccination. Infection and Immunity, 67: 5979-5989.

27. Musher DM, Groover JE, Graviss EA \& Baughn RE (1996). Lack of association between aging and post vaccination levels of lgG antibody capsular polysaccharides of Streptococcus pneumoniae. Clinical Infectious Diseases, 22: 165-167.

28. Musher DM, Groover JE, Rowland JM, Watson DA, Struewing JB, Baughn RE \& Mufson MA (1993). Antibody to capsular polysaccharides of $S$. pneumoniae: prevalence, persistence and response to revaccination. Clinical Infectious Diseases, 17: 66-73.
29. Sankilampi U, Honkanen PO, Bloigu A \& Leinonen M (1997). Persistence of pneumococcal capsular polysaccharide vaccine in the elderly. Journal of Infectious Diseases, 176: 1100-1104.

30. Sorensen RU, Leiva LE, Javier III FC, Sacerdote DM, Bradford N, Butler B, Giangrosso PA \& Moore C (1998). Influence of age on the response to $S$. pneumoniae in patients with recurrent infections and normal immunoglobulin concentrations. Journal of Allergy and Clinical Immunology, 102: 215-221.

31. Conception N \& Frasch C (1998). Evaluation of previously assigned antibody concentrations in pneumococcal polysaccharide reference serum 89-SF by the method of cross-standardization. Clinical and Diagnostic Laboratory Immunology, 5: 199-204.

32. Quataert SA, Kirch C, Diakun K \& Madore DV (1998). Antibody units assigned to anti-pneumococcal serum lot 89-S. In: Program and Abstracts of the International Symposium on Pneumococci and Pneumococcal Disease, Helsinger, Denmark. Streptococcus Unit at Statens Serum Institute, Copenhagen, Denmark, 1998: 67 (Abstract P-28). 